\title{
Measurement of the Weak Mixing Angle in Parity Violating Møller Scattering
}

\author{
Yury G. Kolomensky* ${ }^{\dagger}$ \\ Department of Physics, University of California at Berkeley, Berkeley, CA 94720 \\ E-mail: 'yurugy@physics berkeley.edu'
}

ABSTRACT: We report on the status of the SLAC experiment E158, which plans to measure the value of the electroweak mixing angle $\sin ^{2} \theta_{W}$ at the low $Q^{2}=0.03 \mathrm{GeV}^{2}$ in the polarized Møller scattering with precision of $\sigma\left(\sin ^{2} \theta_{\mathrm{W}}\right)=0.0008$.

\section{Introduction}

Over the past three decades, the Standard Model has been established as the very successful theory describing three of the four fundamental interactions. It has been supported by the wealth of experimental data [i]1] [ Electroweak observables have been measured at the $Z^{0}$ resonance with spectacular precision at CERN and SLAC. However, comprehensive tests of the Standard Model are incomplete without equally precise measurements off the $Z^{0}$ pole. Such measurements, if carried out with sufficient precision, would provide significant constraints on the dynamics of the electroweak interactions at higher orders in the couplings, and could shed light on the new physics beyond the Standard Model at the TeV scales. The physics reach of the low energy parity violation experiments is complimentary to the discovery potential of the high-energy collider experiments.

Elastic $e^{-} e^{-} \rightarrow e^{-} e^{-}$(Møller) scattering has been a clean, well-understood process [2i]. However, the parity non-conservation in Møller scattering, a much more subtle effect which arises due to the interference between the electromagnetic and weak neutral currents, has never been measured before. Experiment E158 [3] will measure the left-right asymmetry $A_{\mathrm{LR}}$ in polarized Møller scattering in the End Station A at SLAC. The goal of E158 is to measure $A_{\mathrm{LR}}$ and determine the value of the weak mixing angle $\sin ^{2} \theta_{\mathrm{W}}$ with a combined precision of 0.0008. Combining the E158 results with the value of the Weinberg angle measured at the $Z^{0}$ pole would establish the running of the weak mixing angle, one of a few Standard Model phenomena still untested by experiment, with $8 \sigma$ significance. Deviations

${ }^{*}$ Speaker.

${ }^{\dagger}$ For the SLAC E158 Collaboration 
from the Standard Model predictions could signal the presence of new physics contributions. E158 results would be sensitive to a wide range of new effects, from new neutral currents mediated by $Z^{\prime}$ bosons in the mass range of 600 to $900 \mathrm{GeV}$, to new contact interactions with a scale of up to 10 to $14 \mathrm{TeV}$.

\section{Parity Violation in Low Energy Møller Scattering}

The spin-averaged differential cross section for Møller scattering is given by

$$
\frac{d \sigma}{d \Omega}=\frac{\alpha^{2}}{2 m E} \frac{\left(3+\cos ^{2} \Theta\right)}{\sin ^{4} \Theta},
$$

where $m$ is the electron mass, $E$ is the beam energy, $\Theta$ is the scattering angle in the centerof-mass frame, and $\alpha$ is the fine structure constant. The total cross section, integrated over the spectrometer acceptance $0.5<\cos \Theta<0$, is approximately $14 \mu$ Barns. The parity violating asymmetry in scattering of polarized electrons off unpolarized targets is defined as

$$
A_{\mathrm{LR}}=\frac{\sigma_{\mathrm{R}}-\sigma_{\mathrm{L}}}{\sigma_{\mathrm{R}}+\sigma_{\mathrm{L}}}
$$

where $\sigma_{\mathrm{R}}\left(\sigma_{\mathrm{L}}\right)$ is the scattering cross section for the longitudinally polarized right(left)handed electrons. The nonzero asymmetry arises from the interference between the treelevel $\gamma$ and $Z^{0}$ exchange amplitudes, and is proportional to their ratio. The tree-level Standard Model asymmetry is given by [i $\left[\begin{array}{l}\overline{4} \\ {[}\end{array}\right]$

$$
A_{\mathrm{LR}}=\left|\frac{A_{Z}}{A_{\gamma}}\right|=m E \frac{G_{F}}{\sqrt{2} \pi \alpha} \frac{16 \sin ^{2} \Theta}{\left(3+\cos ^{2} \Theta\right)^{2}}\left(\frac{1}{4}-\sin ^{2} \theta_{W}\right)
$$

where $G_{F}$ is the Fermi constant. At the $Q^{2}$ of $0.03 \mathrm{GeV}^{2}$, the tree-level asymmetry $A_{\mathrm{LR}}=$ $270 \cdot 10^{-9}$.

At the one-loop level, the electroweak radiative corrections increase the effective value of $\sin ^{2} \theta_{\mathrm{W}}$ by about $3 \%$, reducing Møller asymmetry by $38 \%$ [i]. Beam polarization of $P_{b} \approx$ $80 \%$ and a small dilution from background processes further decrease the raw experimental asymmetry to the level of approximately 120 parts per billion (ppb), which would be measured to the relative precision of about $10 \%$, resulting in the uncertainty on on $\sin ^{2} \theta_{\mathrm{W}}$ of $\sigma\left(\sin ^{2} \theta_{\mathrm{W}}\right)= \pm 0.0007$ (stat.) $\pm 0.003 \quad$ (syst.). The presently available experimental data and the projected E158 uncertainty on $\sin ^{2} \theta_{\mathrm{W}}$ are summarized in Fig. 高

\section{Experimental apparatus}

The experiment consists of scattering of longitudinally polarized high energy electrons off atomic electrons in an unpolarized liquid hydrogen target. The incident electrons are accelerated in 200-300 ns long pulses through the 2-mile SLAC linac to 45 or $48 \mathrm{GeV}$ and delivered to the End Station A. The helicity of the polarized beam is flipped pseudorandomly (i.e., according to a pre-defined and reproducible random number algorithm) at the electron source with $120 \mathrm{~Hz}$ frequency on a pulse-by-pulse basis. The scattered electrons are transported through a magnetic spectrometer (Fig. $\overline{2}$, in $)$, consisting of three dipole and 
four quadrupole magnets. The spectrometer is designed to focus the Møller electrons in a small ring $60 \mathrm{~m}$ downstream of the target, while spreading the particles from $e p$ interactions over a larger area and shielding the detectors from the hard photons produced in the target. The electromagnetic calorimeter integrates the response of $(3-4) \cdot 10^{7}$ scattered electrons per pulse. It consists of quartz fibers sandwiched between layers of copper, for a total thickness of about 15 radiation lengths. The design possesses excellent radiation hardness (>1 GRad) while providing modest energy resolution (10\%).

The primary physics backgrounds in the experiment are electrons scattered elastically and inelastically off target protons, and charged hadron $\$ 236$ photo-produced at the target. The rate of both backgrounds has been estimated using GEANT [īin] Monte Carlo simulation, and corresponds to $10-20 \%$ of the Møller rate. The rate and asymmetry of both types of backgrounds will be measured using additional detectors. The elastic and inelastic ep background will be measured with a calorimeter place at a large distance from the beam pipe, outside of the Møller peak.

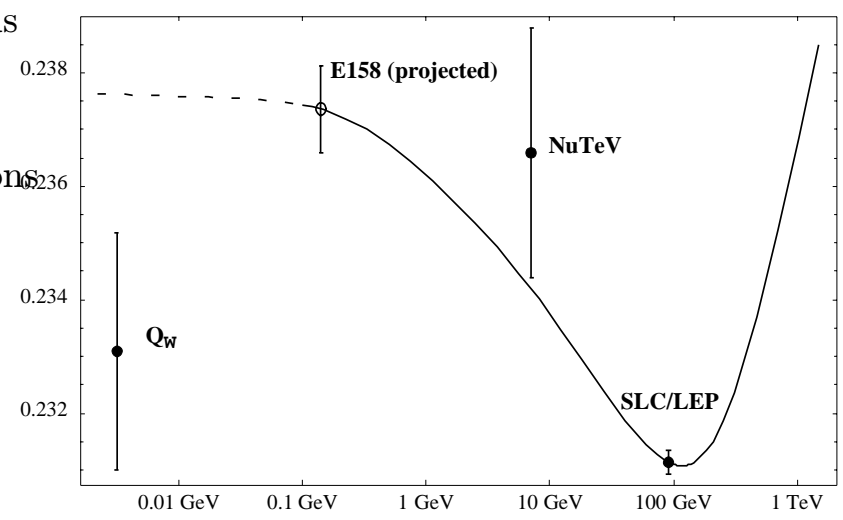

Figure 1: The compilation of data on $\sin ^{2} \theta_{\mathrm{W}}$ and the projected experimental precision of E158. $Q_{W}$ refers to the measurement of the parity violation in atomic Cs ["6.], and $\mathrm{NuTeV}$ result comes from [7]. Also shown is a combined result for $\sin ^{2} \theta_{\mathrm{W}}$ from the $Z^{0}$ pole observables [i]

The charged hadron asymmetry will be measured using a special pion detector, placed behind the Møller detector and an absorber wall.

Additionally, a forward-angle luminosity monitor is placed at small distance from the beam axis, downstream of the main detector. The small-angle ep and Møller scattering provide high counting rates and possess small Standard Model asymmetry. The detector is designed to be sensitive to changes in the instantaneous luminosity at the $10^{-4}$ level, and thus can be used to monitor changes in target density, e.g. due to boiling of the liquid hydrogen. It will also provide a strong check of systematic effects by measuring null asymmetry with a statistical accuracy better than that of the main Møller detector.

\section{Beam-related Systematics}

One of the most important challenges for the experiment is controlling the systematic effects related to the polarized beam. The incident electron helicity is flipped very rapidly, and hence slow effects such as changes in spectrometer acceptance, diurnal pedestal or calibration drifts, etc. are not very dangerous. However, in order to measure a small physics asymmetry with a part-per-billion precision, pulse-to-pulse variations in the beam properties need to be carefully monitored and controlled. The residual asymmetry in the incident beam charge, position, or energy can be introduced by non-uniformities in the strained GaAs cathode or linear polarization component in the laser light at the polarized 


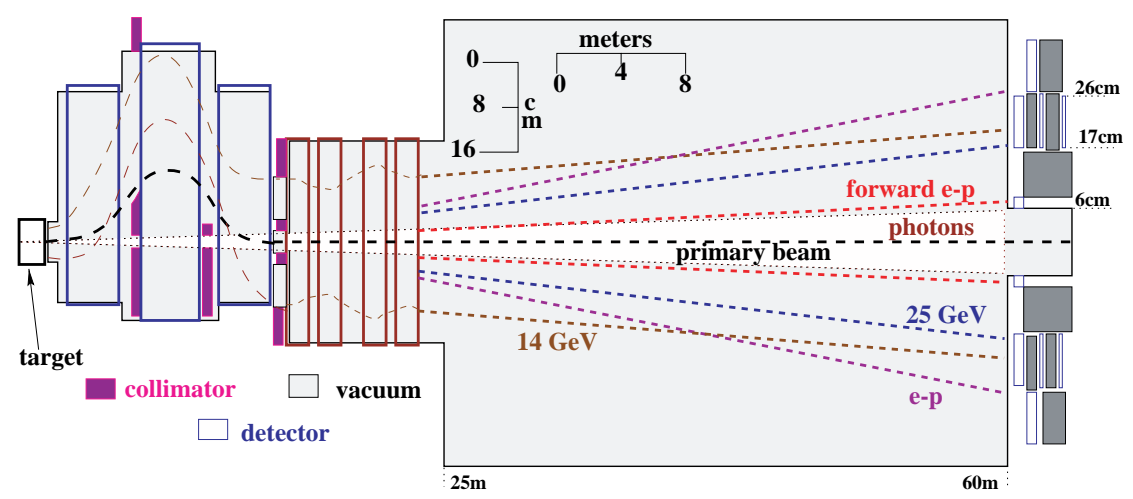

Figure 2: The layout of E158 spectrometer.

electron source [1] $\left.{ }_{1}^{1} \overline{0}\right]$, or it can be statistical in nature, and arise from non-perfect cancellation between average beam position and charge for left- and right-handed pulses. We plan to correct for these effects by precisely measuring the beam charge and position for every pulse, and by applying fast feedbacks [i] $[1]$ in order to zero the residual asymmetries. Additional cancellations of false asymmetries and systematic checks will come from the periodic passive reversals of the laser light helicity using half-wave plates at the source. We also plan to run at two beam energies of 45 and $48 \mathrm{GeV}$. Each energy corresponds to an integer number of $g-2$ precessions of electron spin in the $24.5^{\circ}$ A-Line bend. Thus, each energy corresponds to the different orientation of the electron helicity at the target relative to the laser light polarization. Source systematic effects, sensitive to the laser light helicity, will largely cancel out when the two data sets are added.

The integrated beam charge will be measured each pulse using a number of toroidal magnetic beam current transformers (toroids), equipped with low noise readout electronics and custom 16-bit integrating ADCs. The beam position, angle, and energy will be measured by resonant microwave BPMs [1, $\left[\begin{array}{l}1 \\ -1\end{array}\right]$, equipped with custom RF electronics with I/Q demodulation [i] $[\overline{1} \overline{3}]$.

\section{Status and Prospects}

We had tested all major subsystems of the experiment in a series of test runs, before E158 has been scheduled to take physics data in the Spring of 2002. The beam instrumentation hardware was tested in a January 1999 and November 2000 runs, and demonstrated the beam position resolution of $500 \mathrm{~nm}$ and charge resolution of $3 \cdot 10^{-5}$ per pulse. We have also successfully commissioned the beam asymmetry feedback systems, demonstrating that the false charge asymmetry can be maintained at the level of $200 \mathrm{ppb}$ for a week-long run [i] 1 in. Position asymmetry was maintained at $10-20 \mathrm{~nm}$ level for the same time period. Both benchmarks are within the experimental requirements, and demonstrate that under active feedbacks the false beam asymmetries average out to zero faster than the statistical $1 / \sqrt{N}$ law. Performance of the beam monitors and asymmetry feedbacks is demonstrated in Fig. $\overline{\beta_{1}} \cdot$ 

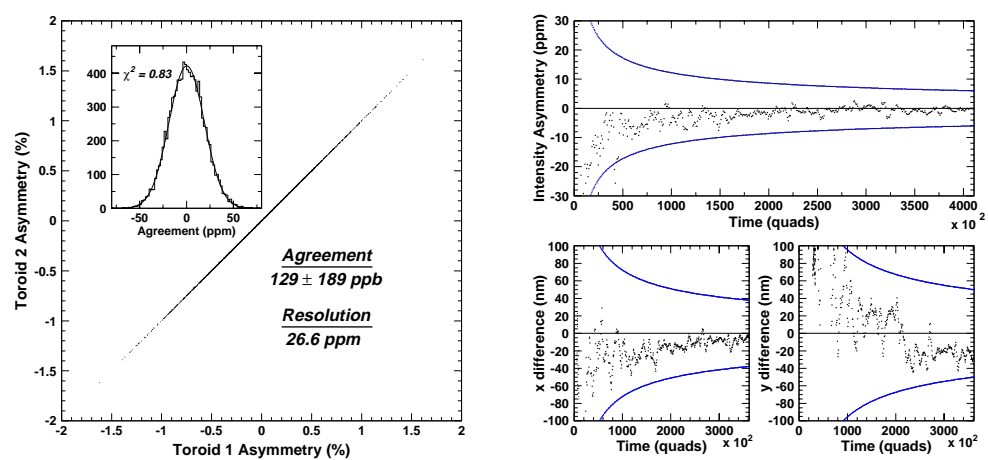

Figure 3: Resolution of the beam instrumentation devices and performance of asymmetry feedback. Shown is the pulse-to-pulse agreement between the asymmetries measured in two toroids (left), showing the average agreement of $1.3 \cdot 10^{-7}$, dominated by statistics, and the pulse-to-pulse resolution of $2.7 \cdot 10^{-5}$. On the right plot are the results of the 2 hour asymmetry feedback run showing integrated charge (top) and position (bottom) asymmetries as a function of time. Curves represent statistical asymmetry bound of $1 / \sqrt{N_{\text {pulses }}}$.

The spectrometer and detector construction and installation was completed in the Winter 2001, and all detector systems have been operational in the Spring 2001 engineering run. Some problems were uncovered, in particular, significant fluctuations in the number of hard background photons, originating in the target and bouncing off the collimator apertures. These problems are being addressed before the start of the commissioning run in February 2002. The experiment is on track to take the first physics data in April and May 2002.

\section{References}

[1] D. Charlton, in these proceedings.

[2] C. Møller, Ann. Phys. 14, 531 (1932) .

[3] The E158 Collaboration, R. Carr et al., SLAC-Proposal-E-158 (1997).

[4] E. Derman and W.J. Marciano, Ann. Phys. 121, 147 (1979) .

[5] A. Czarnecki and W.J. Marciano, Phys. Rev. D53, 1066 (1996) .

A. Czarnecki and W.J. Marciano, Int. J. Mod. Phys. A15, 2365 (2000) .

[6] C.S. Wood et al., Science 275, 1759 (1997) .

[7] K.S. McFarland et al., Eur. Phys. Jour. C31, 509 (1998) .

[8] D.E. Groom et al. (Particle Data Group), The European Physical Journal C15, 1 (2000).

[9] CERN Program Library Long Writeup W5013, CERN Geneva, Switzerland (1993).

[10] R. Alley et al., Nucl. Instrum. Methods A365, 1 (1995) .

[11] P. Mastromarino et al., to be published in IEEE Trans. on Nucl. Sci.

[12] Z.D. Farkas et al., preprint SLAC-PUB-1823 (1976).

[13] D.H. Whittum, Yu.G. Kolomensky, Rev. Sci. Instrum. 70, 2300 (1999) . 